\title{
Easy and straightforward construction of wideband phase-shifting algorithms for interferometry
}

\author{
J. C. Estrada, ${ }^{1, *}$ M. Servin, ${ }^{1}$ and J. A. Quiroga ${ }^{2}$ \\ ${ }^{1}$ Centro de Investigaciones en Óptica Asociacion Civil, León Guanajuato 20036, México \\ ${ }^{2}$ Departamento de Óptica, Universidad Complutense de Madrid, Ciudad Universitaria S/N, 2841 Madrid, Spain \\ *Corresponding author: jcesar_estrada@hotmail.com
}

Received October 22, 2008; revised December 18, 2008; accepted December 19, 2008; posted January 7, 2008 (Doc. ID 103049); published February 5, 2009

We show a practical way for building wideband phase-shifting algorithms for interferometry. The idea presented combines first- and second-order quadrature filters to obtain wideband phase-shifting algorithms. These first- and second-order quadrature filters are analogous to the first- and second-order filters commonly used in communications engineering, named building blocks. We present a systematic way to develop phaseshifting algorithms with large detuning robustness or large bandwidth. In general, the approach presented here gives a powerful frequency analysis and design tool for phase-shifting algorithms robust to detuning for interferometry. (C) 2009 Optical Society of America

OCIS codes: $120.3940,120.3180,120.2650,120.5050$

In this work, we present an easy and straightforward technique for designing temporal phase-shifting (TPS) algorithms with large detuning [1]. It is wellknown that TPS algorithms may be regarded also as quadrature filters tuned at a single temporal frequency. The tuning frequency is the temporal carrier of interferograms, which in TPS interferometry parlance is the phase step used to obtain the interferograms. Here we have adopted a filter construction strategy typically followed in communications engineering, which is to build larger order quadrature filters based upon simpler building blocks, namely, first- and second-order filters. The main advantage of adopting this strategy is that these lower order discrete quadrature filters may be optimally located in the frequency space to obtain a quadrature filter with large detuning robustness (large bandwidth).

In interferometry, interferometric data are obtained as an image called an interferogram. The temporal sampling of interferometric data at a site $(x, y)$, can be modeled as a periodical signal $s: \mathbb{R} \rightarrow \mathbb{R}$ in the following way:

$$
s(t)=a_{x, y}+b_{x, y} \cos \left(\phi_{x, y}+\omega_{0} t\right), \quad t \in \mathbb{I},
$$

where $a_{x, y} \in \mathbb{R}$ and $b_{x, y} \in \mathbb{R}$ are the dc and contrast term at site $(x, y)$, respectively. $\phi_{x, y} \in \mathbb{R}$ is the unknown phase in that site, and $\omega_{0} \in \mathbb{R}$ is the linear phase shifting or temporal frequency carrier; $t$ is the temporal sampling. The problem here is the following: given a signal like the one shown in Eq. (1), find a way to recover the unknown phase $\phi_{x, y}$. On this topic we can find several works that deal with this problem, to mention some of them we can cite [2-10]. Taking a look at previous works around linear phaseshifting algorithms, we can see that most of the algorithms were developed intuitively, or systematically using least squares $[2,4,5]$. Actually, to our knowledge the only work to describe the phase-shifting algorithms in the Fourier domain is that published by Freishlad and Koliopoulos [11]. Here, we will show how to design phase-shifting algorithms in the Fourier domain.
As we said at the beginning, for the TPS demodulation problem we have adopted a filter construction strategy based on simple building blocks or filters. Then, let us start by defining the following first- and second-order basic building blocks:

$$
\begin{aligned}
& h_{1}(t)=\frac{i}{2}[\delta(t-1)-\delta(t+1)], \\
& h_{2}(t)=2 \delta(t)-\delta(t-1)-\delta(t+1),
\end{aligned}
$$

which are a first- and second-order difference operator, respectively, where $\delta(t)$ is the Dirac delta function, and $i=\sqrt{-1}$. These filters have as a frequency response the following expressions:

$$
\begin{aligned}
& H_{1}(\omega)=-\sin (\omega), \\
& H_{2}(\omega)=2-2 \cos (\omega) .
\end{aligned}
$$

These building blocks, can be used as first- and second-order quadrature filters, respectively. To show this, take a look at Fig. 1. There, we show the graphics of these difference operators or building blocks in the Fourier domain. In Fig. 1(a), we show the graphic of Eq. (4), and in Fig. 1(c), we show the graphic of Eq. (5). As these building blocks remove the frequency component at $\omega=0$, we see these building blocks as

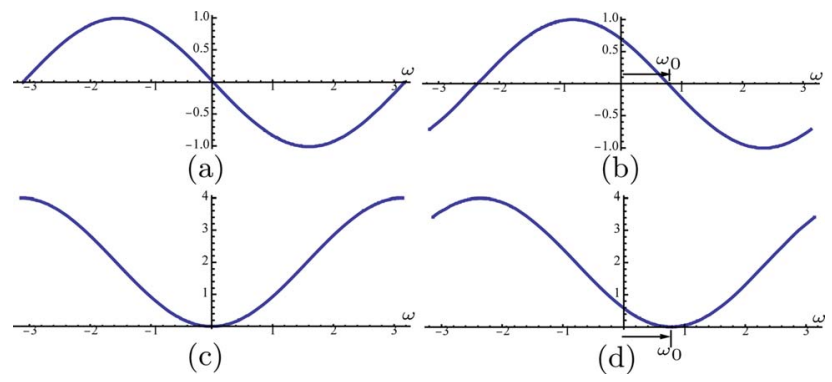

Fig. 1. (Color online) (a) shows the graphic of Eq. (4), and (b) shows the graphic of Eq. (5). (c) and (d) show the graphics of Eqs. (4) and (5), shifted (tuned) at $\omega=\omega_{0}$, respectively. 
high-pass filters. On the other hand, if we shift these building blocks to $\omega=\omega_{0}$, as shown in Figs. 1(b) and $1(\mathrm{~d})$, these building blocks remove the frequency component at $\omega=\omega_{0}$. Therefore, we see the shifted building blocks of Figs. 1(c) and 1(d) as quadrature filters for signals $s: \mathbb{R} \rightarrow \mathbb{R}$ given as $s(t)=b \cos \left(\phi+\omega_{0} t\right)$, $\forall\left(\phi, \omega_{0}\right) \in \mathbb{R}$. That is, if our signal is given as $s(t)$ $=b \cos \left(\phi+\omega_{0} t\right), \forall\left(\phi, \omega_{0}\right) \in \mathbb{R}$, using a filter shifted (tuned) to $\omega=\omega_{0}$, like that given in Figs. 1(b) and 1(d), one obtains an analytical signal $s^{\prime}: \mathbb{R} \rightarrow \mathrm{C}$ given as $s^{\prime}(t)=C \exp \left[i\left(\phi+\omega_{0} t\right)\right]$, where constant $C \in \mathbb{R}$ has to be with the quadrature filter response. In this way, the reader can see the building blocks of Eqs. (4) and (5) as first- and second-order quadrature filters, respectively; however, in this Letter we indistinctly call these filters building blocks, because they are used to build more complex filters, as in communications engineering, to obtain wideband phase-shifting algorithms.

Using the building blocks shown in Eqs. (4) and (5), we can obtain linear phase-shifting algorithms for interferometry. As we said before, the TSP algorithms may be regarded as quadrature filters tuned at a single temporal frequency, that is, a quadrature filter that obtains as a result an analytical signal given as $s^{\prime}(t)=C \exp \left[i\left(\phi_{x, y}+\omega_{0} t\right)\right]$, from a real signal like that shown in Eq. (1). For example, a five-step phaseshifting algorithm can be obtained by combining a first-order building block centered at $\omega=0$ to remove the dc term $a$ from Eq. (1), and a second-order building block shifted (tuned) at $\omega=\omega_{0}$ in the following way:

$$
H(\omega)=H_{1}(\omega) H_{2}\left(\omega-\omega_{0}\right)=-\sin (\omega)\left[2-2 \cos \left(\omega-\omega_{0}\right)\right]
$$

In Fig. 2, we can see the graphic of this quadrature filter obtained by using the building blocks of Eqs. (4) and (5). In Fig. 2(a), we show graphically how the first- and second-order basic building blocks in the Fourier domain are located apart, while in Fig. 2(b) we show the quadrature filter obtained as the product of these basic building blocks. There, we can see that this obtained quadrature filter removes both the dc term at $\omega=0$ and the frequency component at $\omega$ $=\omega_{0}$, which is the tuning frequency. Now, taking the inverse Fourier transform of the quadrature filter shown in Eq. (6) we obtain the following convolution operator for a five-step phase-shifting algorithm:

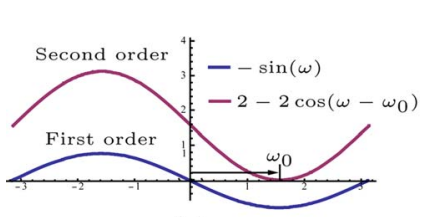

(a)

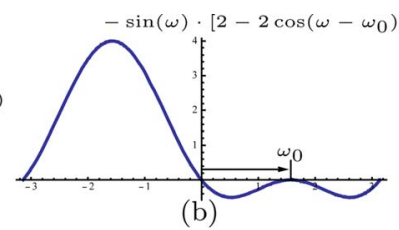

(b)
Fig. 2. (Color online) (a) shows the first- and second-order building block filters, and (b) shows the product of these building blocks. We can see in (b) that the quadrature filter obtained removes the dc term at $\omega=0$ and the frequency component at $\omega=\omega_{0}$.

$$
\begin{aligned}
h(t)= & {[2 \delta(t)-\delta(t-2)-\delta(t+2)] \sin \left(\omega_{0}\right) / 2+i[2 \delta(t-1)} \\
& -2 \delta(t+1)] / 2-i[\delta(t-2)-\delta(t+2)] \cos \left(\omega_{0}\right) / 2 .
\end{aligned}
$$

As we want the mathematical formula of the five-step phase-shifting algorithm, it is necessary convolve this operator with our interferometric signal of Eq. (1) and take its angle at $t=0$. Making this, we obtain the following formula to recover the phase from a temporal interferogram phase-shifted sequence:

$$
\phi_{x, y}=\tan ^{-1}\left[\frac{2 s(-1)-2 s(1)-[s(-2)-s(2)] \cos \left(\omega_{0}\right)}{[2 s(0)-s(-2)-s(2)] \sin \left(\omega_{0}\right)}\right],
$$

from which, taking the particular case $\omega_{0}=\pi / 2$, one obtains

$$
\phi_{x, y}=\tan ^{-1}\left[\frac{2 s(-1)-2 s(1)}{2 s(0)-s(-2)-s(2)}\right],
$$

which is the classic Hariharan five-step phaseshifting algorithm [10]. Thus, we can see that the quadrature filter approach presented here is consistent with the already developed phase-shifting algorithms as particular cases. However, the approach presented here is more powerful, since we can design general phase-shifting algorithms in a more easy and straightforward way than by using the least-squares approach (see [4,6]) for any arbitrary phase step $\omega_{0} \in \mathbb{R}$.

Now, let us show how this powerful construction tool can generate what we call wideband phaseshifting algorithms. Again, for illustration purposes, let us show it first in a graphic picture. Taking a look at Fig. 3(a), we use a first-order quadrature filter located at $\omega=0$ and three second-order quadrature filters located at $\omega_{0}=\pi / 4, \omega_{1}=\pi / 2$, and $\omega_{2}=3(\pi / 4)$. Then, taking the product of these quadrature filters, we obtain the wideband quadrature filter shown in Fig. 3(b), whose tuning range is almost between 0 and $\pi \mathrm{rad}$. Mathematically, the quadrature filter of Fig. 3(b) is the following:

$$
H(\omega)=H_{1}(\omega) H_{2}\left(\omega-\omega_{0}\right) H_{2}\left(\omega-\omega_{1}\right) H_{2}\left(\omega-\omega_{2}\right),
$$

where $H_{1}\left(\right.$ ) and $H_{2}($ ) are the first- and second-order quadrature filters from Eqs. (4) and (5). Now, taking the inverse Fourier transform of Eq. (10), we have the following:

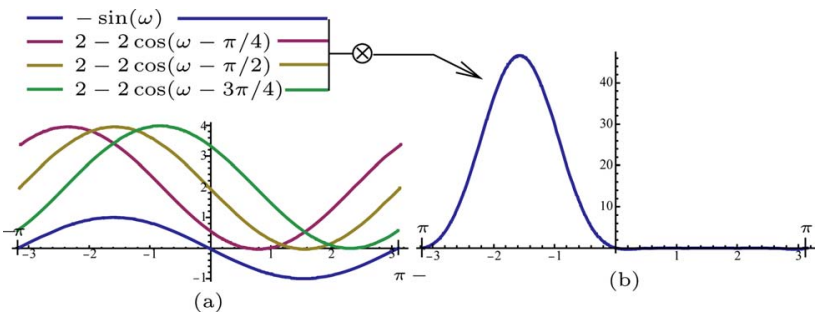

Fig. 3. (Color online) (a) shows the frequency locations of the basic building blocks used to construct the wideband quadrature filter shown in (b). The obtained quadrature filter is the product of these building blocks. 


$$
\begin{aligned}
h(t)= & \frac{1}{2} \delta(t-4)-(2 \sqrt{2}+3) \delta(t-2)+(4 \sqrt{2}+5) \delta(t) \\
& -(2 \sqrt{2}+3) \delta(t+2)+\frac{1}{2} \delta(t+4)-i[(\sqrt{2}+1) \delta(t-3) \\
& -(3 \sqrt{2}+5) \delta(t-1)+(3 \sqrt{2}+5) \delta(t+1) \\
& -(\sqrt{2}+1) \delta(t+3)] .
\end{aligned}
$$

Finally, to obtain the mathematical formula for the wideband phase-shifting algorithm we convolve this quadrature filter with the signal of Eq. (1) and take its angle at $t=0$. Then, we obtain the following ninestep wideband phase-shifting algorithm:

$$
\phi_{x, y}=\tan ^{-1}\left[\frac{(\sqrt{2}+1) s(-3)-(3 \sqrt{2}+5) s(-1)+(3 \sqrt{2}+5) s(1)-(\sqrt{2}+1) s(3)}{\frac{1}{2} s(-4)-(2 \sqrt{2}+3) s(-2)+(4 \sqrt{2}+5) s(0)-(2 \sqrt{2}+3) s(2)+\frac{1}{2} s(4)}\right] .
$$

Now, let us test it with a simulated signal whose frequency is between 0 and $\pi \mathrm{rad}$. In Fig. 4 we show this phase estimation test. Figure 4(a) shows the ground true phase used to generate the signal. If we take its derivative, we can see that its frequency is 0 for $t$ $=0$ and $\pi$ for $t=256$. Figure 4(b) shows the signal under test whose phase is shown in Fig. 4(a). It is necesary to remark that the presentation of the signal's graphic suffers from aliasing owing to the limited resolution of the software used to generate the graphics. Given this signal, we estimated its phase with Eq. (12), and in Fig. 4(c) we graph the absolute phase error of the estimated phase, that is $\left|\phi_{x, y}(t)-\hat{\phi}_{x, y}(t)\right|$, where $\phi_{x, y}(t)$ is the ground true phase and $\hat{\phi}_{x, y}(t)$ is
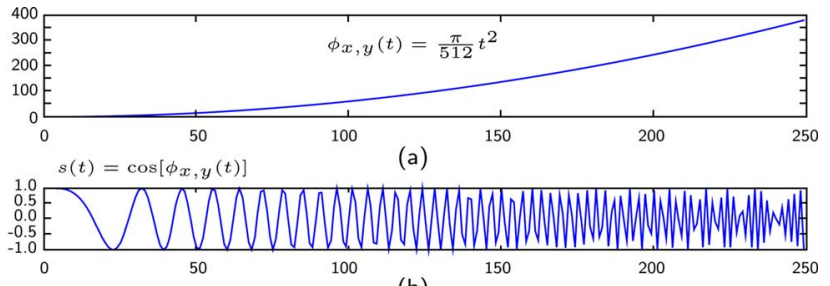

(b)

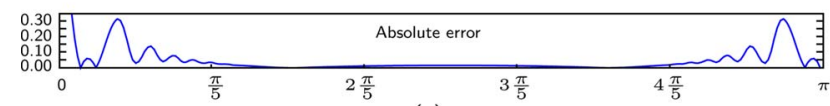

(c)

Fig. 4. (Color online) (a) is the ground true phase, (b) is the generated signal, and (c) is the absolute phase error of estimated phase using the wideband phase-shifting algorithm of Eq. (12). Note: The presentation of graphic (b) suffers from aliasing owing to the limited resolution of the software used to generate the graphics. the estimated phase previously unwrapped. There we can see that the absolute phase error close to the frequencies 0 and $\pi$ is almost $0.3 \mathrm{rad}$, and at least zero between frequencies $\pi / 5$ and $4(\pi / 5)$. This is expected if we see the frequency response of the algorithm used in Fig. 3.

Summing up, the quadrature filter approach presented here is very useful for both describing phaseshifting algorithms in the Fourier domain and generating new phase-shifting algorithms with the desired bandwidth.

\section{References}

1. D. Malacara, M. Servin, and Z. Malacara, Interferogram Analysis for Optical Testing (Marcel Dekker, 1998).

2. J. H. Bruning, D. R. Herriott, J. E. Gallagher, D. P. Rosenfeld, A. D. White, and D. J. Brangaccio, Appl. Opt. 13, 2693 (1974).

3. J. C. Wyant, Appl. Opt. 14, 2622 (1975).

4. C. J. Morgan, Opt. Lett. 7, 368 (1982).

5. J. Schwider, R. Burow, K.-E. Elssner, J. Grzanna, R. Spolaczyk, and K. Merkel, Appl. Opt. 22, 3421 (1983).

6. J. E. Greivenkamp, Opt. Eng. (Bellingham) 23, 350 (1984).

7. Y.-Y. Cheng and J. C. Wyant, Appl. Opt. 24, 3049 (1985).

8. C. Ai and J. C. Wyant, Appl. Opt. 26, 1112 (1987).

9. P. Hariharan, Appl. Opt. 26, 2506 (1987).

10. P. Hariharan, B. F. Oreb, and T. Eiju, Appl. Opt. 26, 2504 (1987).

11. K. Freishlad and C. L. Koliopoulos, J. Opt. Soc. Am. A 7, $542(1990)$. 